\title{
EL GÉNERO ISISTIUS (SQUALIDAE) EN EL ALTO GUAYACÁN. FORMACIÓN USCARI (MIOCENO SUPERIOR-PLIOCENO INFERIOR), PROVINCIA DE LIMÓN, COSTA RICA
}

\author{
César A. Laurito M. \\ Sección de Paleontología y Estratigrafía, Dirección de Geología y Minas; \\ Ministerio del Ambiente y Energía. Apartado postal 10104-1000, \\ San José, Costa Rica
}

(Recibido 6/8/1996; Aceptado 13/3/1997)

\begin{abstract}
Excavations in the Caribbean region of Costa Rica from the upper Miocene Alto Guayacán beds, are yelding a diverse sharks fauna. Several of the teeth thus far recovered are referred to Isistius triangulus, and are the second found recorded of this species in the New World. Detailled descriptions of the micro-teeth morphology and paleoecological inference are given.

RESUMEN: Se hace una descripción topológica de piezas dentales correspondientes a tiburones del género Isistius, conocidos actualmente como tollos cigarros. Estos fueron recuperados del afloramiento de Alto Guayacán del Mioceno superior terminal-Plioceno Inferior basal de la Provincia de Limón, en el Caribe costarricense.
\end{abstract}

\section{INTRODUCCIÓN}

La fauna de tiburones fósiles de Alto Guayacán, represantada principalmente por dientes y raramente por otros elementos óseos como vertebras, es sumamente diversa y abundante. Aguilar (1994), estudió la fauna malacológica y además hizo algunas anotaciones acerca de la fauna de seláceos encontrados en éstos sedimentos, su edad y ambiente basándose principalmente en moluscos.

El objetivo de ésta comunicación es dar a conocer la presencia del género Isistius en la asociación de seláceos fósiles de Alto Guayacán, su importancia biogeográfica, paleoambiental y dar una descripción odontológica del material encontrado.

El material fué colectado de una única localidad, ubicada en las siguientes coordenadas 585,650 W. y 225,350 N. Hoja 3446II Bonilla; escala 1:50,000 del IGNCR (Fig. 1); y se encuentra depositado en la Dirección de Geología y Minas, colección del autor bajo el código CLM-000.

\section{METODOLOGÍA}

La mayoría de los dientes fueron obtenidos directamente del afloramiento. Otros fueron entresacados bajo el microscópio estereoscópico, a partir de un concentrado fosilífero preparado.

El concentrado fue el producto del desagregado de una muestra de sedimento, lavada con abundante agua y tamizada. Sus reciduos fueron secados por cerca de tres horas a temperaturas no mayores de $70^{\circ} \mathrm{C}$. Finalmente, los dientes recuperados fueron lavados en una cubeta ultrasónica, para desprender residuos de arcilla y clastos diversos. 


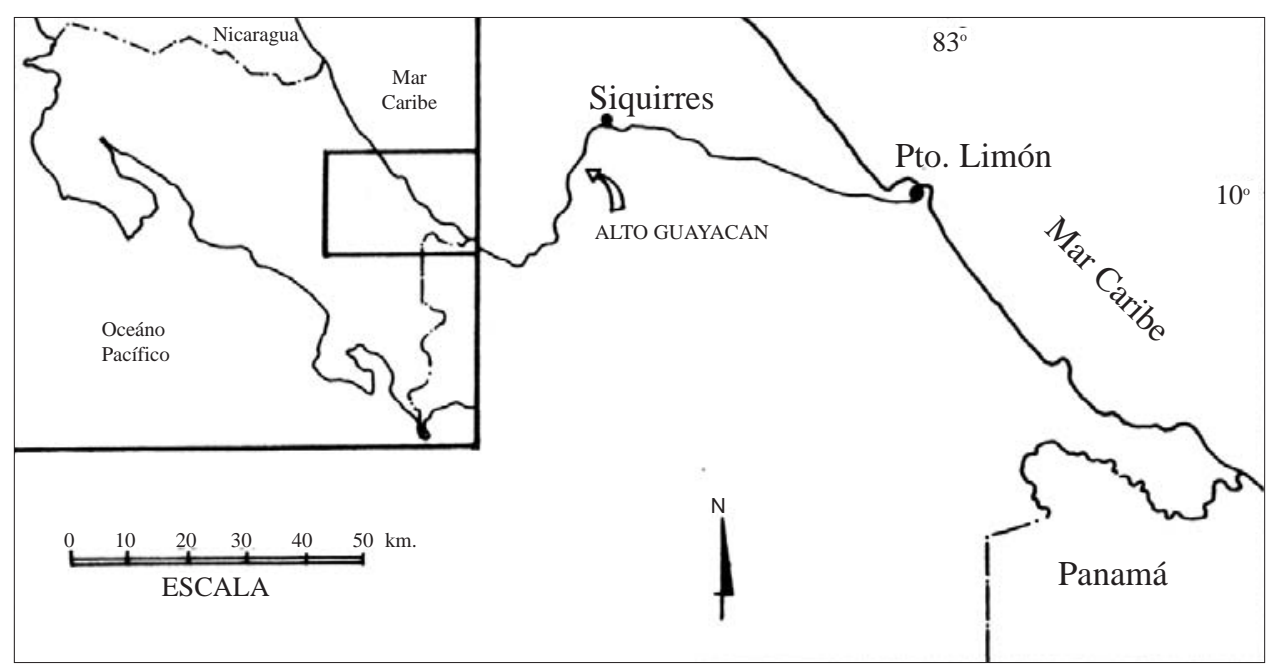

Fig. 1: Ubicación de la localidad de alto Guayacán.

\section{PALEONTOLOGÍA}

La clasificación sigue la sistemática propuesta por Compagno (1984) y Cappetta (1987), descrita a continuación:

Clase Chondrichthyes Huxley 1880

Subclase Elasmobranchii Bonaparte 1838

Cohorte Euselachii Hay 1922

Superorden Squalomorphii Compagno 1973

Orden Squaliformes Goodrich 1929

Familia Squalidae Bonaparte 1834

Subfamilia Dalatiinae Gray 1851

Género Isistius Gill 1864

(Paleoceno Superior a la actualidad)

Especie tipo: Scymmus brasiliensis Quoy y Gaimard 1824

\section{Isistius triangulus (Probst 1879)}

Sinonimia: del género ver Compagno (1984, pág.93), de la especie:

- Scymnus lichia Cuvier in Zbyszewski \& Almeida, 1950, p. 353; lám. 7, fig. 144.

- Scymnus lichia Cuvier in Serralheiro, 1954, p. 76, fig. 2; lám. 2, figs. 77-79.

- Isistius triangulus (Probst) in Cappetta, Granier \& Ledoux, 1967, p. 292; lista.
- Isistius triangulus (Probst) in Cappetta \& Ledoux, 1970 , p. 22.

- Isistius triangulus (Probst) in Cappetta, 1970, p. 74; lám. 8, figs. 3-7.

- Isistius triangulus (Probst) in Antunes \& Jonet, 1970, p. 209, fig. 9; lám. 20, fig. 145.

- Isistius triangulus (Probst) in Ledoux, 1972 , p. 161, fig. 13a-c.

- Isistius triangulus (Probst) in Longbottom, 1979 , p. 65; figs. 25 y 26.

- Isistius triangulus (Probst) in Cappetta, 1987, p. 62; figs. 59 Ia-c.

El género Isistius presenta dentición dignática, es decir los dientes superiores e inferiores son marcadamente diferentes, pero la morfología intra "mandibular" es de tipo monodonta. Los dientes inferiores son típicamnete triangulares y mucho más grandes que los superiores. En terminos generales y de acuerdo con las observaciones de Antunes \& Jonet (1970) y Ledoux (1972), los forámenes de la raíz están dispuestos de igual forma que en Scymnorhinus licha, forma que actualmente habita el mar Mediterráneo.

Por algún motivo que se desconoce, los dientes superiores no se registran en estado fósil, Cappetta (1987) sugiere que esto se debe a que los dientes superiores presentan posiblemente una muy pobre mineralización, lo que provoca 
una selección destructiva de los mismos durante la diagénesis.

\section{MATERIAL: 13 DIENTES}

El CLM-016 corresponde a un diente inferior izquierdo, excepcionalmente bien conservado (Fig. 2a y b) y el CLM-017 corresponde a un diente inferior izquierdo del que se preservó la corona y de manera parcial la raíz. El CLM-018, diente inferior izquierdo con la corona bien preservada y la raíz de manera parcial; el CLM-019 diente inferior derecho bien preservado y el CLM-020 con la corona preservada; CLM-021 diente completo; $C L M-022$ diente con la corona completa y raíz parcial; CLM-023 corona parcial; CLM-024, corona; CLM-025 con la corona bien preservada y la raíz parcial; $C L M-026$ diente comisural izquierdo completo; $C L M$-027 diente izquierdo completo; $C L M-028$ corona preservada.
Todos de color café claro con el esmalte intacto, filos y rebordes bien preservados, sin evidencia de transporte o efecto de dilución gástrica. Lo anterior se evidencia porque los dientes rotos poseen filos no redondeados y no hay dilución del esmalte.

Descripción: diente comprimido en sentido labio-lingual, con la corona en forma de triángulo equilatero y raíz de contorno casi cuadrado. El peto o proceso descendete externo de la corona recubre practicamente la cara labial de la raíz. La corona presenta un aserramiento muy suave, apenas incinuado.

La raíz presenta en su cara externa la endidura labial mediana (Elm) y el ojal (Oj) (Fig. 2a), mientras que la cara lingual presenta el ojal y un poco más arriba el foramen medio lingual (Fml) (Fig. 2b). La raíz da la impresión de ser bífida o bilobada a causa de la endidura labial mediana, que se proyecta hasta alcanzar la parte baja de la corona en su cara labial.
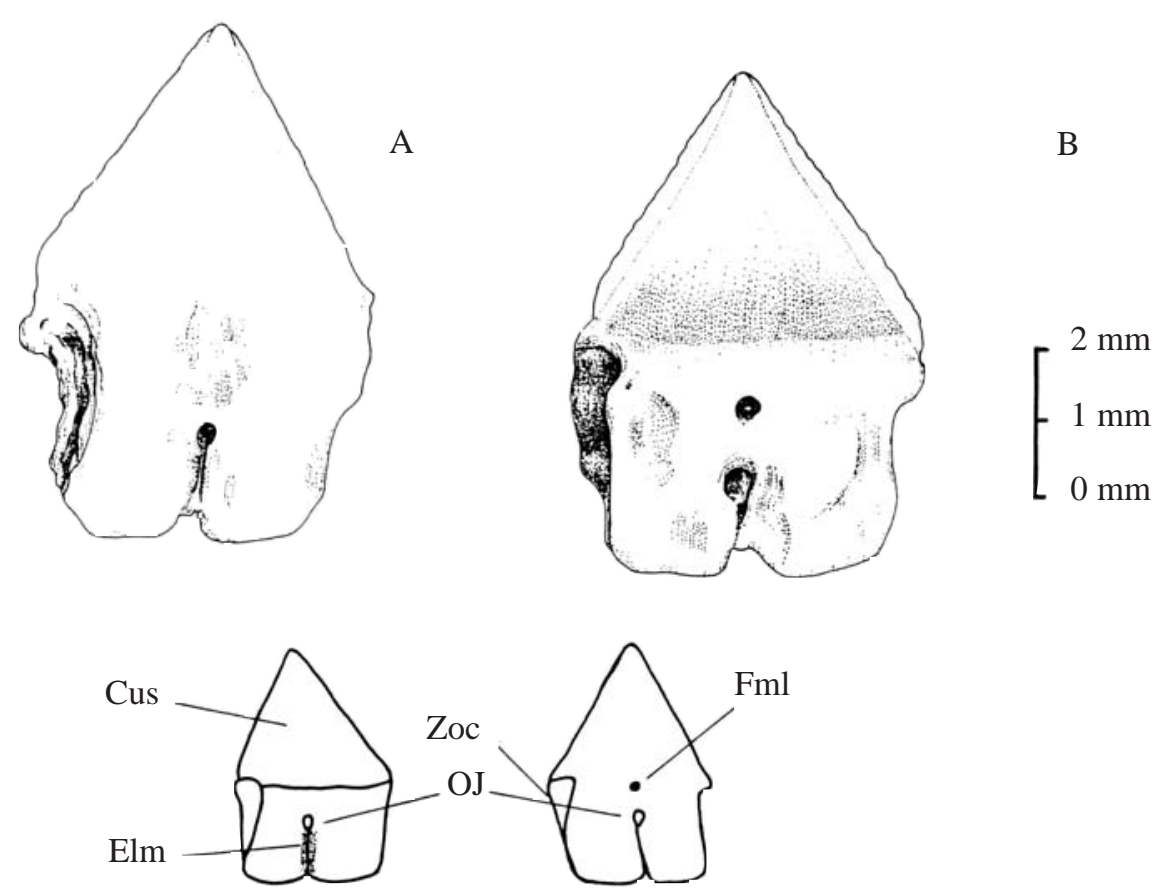

Fig. 2: Diente inferior de Isistius triangulus (a) vista labial, (b) vista lingual; Cus, cúspide; Elm, endidura lingual mediana; Oj, ojal; Zoc, zócalo; Fml, foramen medio lingual. 
Los márgenes mesial y basal de la corona junto con el reborde superior y mesial de la raíz, constituyen una saliencia que se ajusta perfectamente con el zócalo presente en el margen comisural y de posición similar, lo que permite un perfecto ajuste de los dientes entre si, patrón de imbricación descrito por Strasburg (1963).

\section{DISCUSIÓN}

Los dientes inferiores de Isistius triangulus (Probst, 1879), se distinguen de los similares de Isistius brasiliensis (Quoy \& Gaimard, 1824) e Isistius plutodus Garrick \& Springer, 1964; tanto en la forma de la corona, como la configuración de la raíz.

En I. brasiliensis e I. Plutodus la corona se asemeja a un triángulo isóceles, mientras que en la forma fósil el contorno de la corona se aproxima más a un triángulo equilatero. En la forma fósil y las formas actuales, el filo en la corona el aserramiento es laxo, pero visible. En la forma I. plutodus el filo de los dientes es ligeramente sinuoso (cf. Garrick \& Springer, 1964; pág. 680), siendo en $I$. brasiliensis rectilíneo.

Dentro de la especie fósil I. triangulus, es posible encontrar algunos dientes inferiores con filos sinuosos, que le imprimen un aspecto inflado a la corona (ver figs. 9 y 145 de Antunes \& Jonet, 1970) y ejemplares con filos rectos. En Alto Guayacán, ambas morfologías se han encontrado, aunque las formas rectas son las predominantes (ver además las figs. 25 y 26 de Longbottom, 1979 y fig. 13 de Ledoux, 1972; donde se observan las dos posibilidades).

La raíz de la forma fósil tiende a ser más cuadrada, su ancho y altura son muy sinilares al ancho y altura de la corona, mientras que en las formas actuales, la raíz es notablemente rectangular y su altura llega a duplicar la altura de la corona. Esta particularidad se nota más aún en la especie I. plutodus, cuyos dientes son considerablemente mayores que los de la especie I. brasiliensis (ver fig. 2 de Strasburg, 1963).

Por otra parte, el hallazgo de I. triangulus constituye el segundo registro seguro en el Nuevo Mundo, luego de que Longbottom (1979 pág. 66), diera a conocer su hallazgo procedente de los depósitos costeros del Mioceno de Ecuador. Aunque anteriormente, Casier (1958) describió el género Isistius en Barbados, el mismo autor en una publicación posterior (Casier, 1966), redescribe este descubrimiento como Sphyraena kuglieri un grupo de peces óseos. Igualmente Gillette (1984), describe el género Isistius con dudas, basado en supuestos dientes superiores parcialmente preservados; estos fueron encontrados en la Formación Gatún en Panamá. Sin embargo, el hallazgo es muy dudoso, sobre todo porque nunca se han encontrado dientes superiores preservados y es muy probable que este hallazgo corresponda a otro género de seláceo.

\section{HABITAD ACTUAL Y PALEOECOLOGÍA}

El género Isitius, de acuerdo con Compagno (1984), en la actualidad se encuentra representado por dos especies I. brasiliensis e I. plutodus Garrick \& Springer, 1964; este último restringido al sur de Japón y el Golfo de México.

En general son tiburones de pequeño tamaño $(30$ a $40 \mathrm{~cm}$ ), esqueleto sobre calcificado, de amplia distribución oceánica tropical y con rango batimétrico epipelágico hasta batial ( $85 \mathrm{~m}$ a 3500 $\mathrm{m})$. Alcanza las aguas medias $(85 \mathrm{~m})$ durante la noche, lo que implica migraciones verticales de 2000 a 3000 metros diarios.

Tolera vivir en aguas poco oxigenadas, poseen órganos luminosos que recubren totalmente el cuerpo, a excepción de un collar oscuro carente de luminosidad. Su alimentación incluye diversos crustáceos, (copépodos) y calamares, pero puede vivir ectoparasitariamente, alimentandose de grandes peces ósos, tiburones y cetáceos, para ello cuenta con labios succionantes y una faringe modificada para tal fin (cf. Strasburg, 1963 y Compagno, 1985).

Teniendo en cuenta que la morfología dentaría no ha variado practicamente desde el Paleoceno; lo que a su vez ha permitido rastrear el linage del género con bastante certeza desde el Eoceno inferior; más la constante de que sus dientes superiores no se encuentran fósiles; se puede suponer que su modo de vida no ha variado gran cosa por cerca de 35 m.a., en especial por su estilo oportunista de alimentación, su gran adaptabilidad física a los cambios dinámicos de presión y temperatura que muestra la especie actual.

La paleoecología de Isistius ha sido interpretada por diversos autores, siempre teniendo en cuenta la ecología de las formas actuales. Antunes \& Jonet (1970) y Antunes (1972) consideran 
que la batimetría más característica de Isistius es pelágica (profundidades medias) y habitante de aguas tropicales, subtropicales y templadas. Longbottom (1979) lo señala como un habitante típico del ambiente pelágico y poco común del ambiente batial, siempre indicador de aguas tropicales y subtropicales.

\section{PALEOBIOGEOGRAFÍA Y EDAD}

La edad de los sedimentos de Alto Guayacán, corresponden al Mioceno superior terminal, límite Messiniano-Zancleano o zona de foraminíferos plantónicos N-17-19 (Pizarro, D. com.. escrita)

Ictiolitos asociados al género Isistius han sido registrados en los pozos 417 y 418 del trayecto 51 al 53 del Deep Sea Drilling Project, en el Océano Pacífico. Los ictiolitos se componen de dentículos dérmicos, asociados a sedimentos pelágicos, con rangos de distribución en las muestras Eoceno Superior-Mioceno Superior (Kosarek \& Orr, 1980).

La especie I. triangulus, se ha registrado en el Mioceno inferior de Baltringen, Alemania (Probst, 1879 en Cappetta, 1987); Mioceno medio de Portugal (Zbyszewski \& Moitinho D'Almeida, 1950; Serralheiro, 1954 y Antunes \& Jonet, 1970), sur de Francia (Cappetta, et. al, 1967; Cappetta \& Ledoux; 1970, Cappetta, 1970 y Ledoux, 1972), Suiza (Leriche, 1927 en Cappetta, 1987), Ecuador (Longbottom, 1979) y en el Plioceno de Bélgica (Herman 1974b en Cappetta, 1987).

Hasta la fecha no se ha registrado ésta especie en ningún otro sitio del Caribe, ni del continente Americano, a excepción de Ecuador y Costa Rica.

\section{EVOLUCIÓN}

De acuerdo con Cappetta (1987) el género ha cambiado poco a lo largo del tiempo y el linage I. trituratus-I. triangulus-I. brasiliensis es probable rastrearlo con seguridad desde el Eoceno al presente.

Isistius triangulus (Probst, 1879), se distingue de su ancestro I. trituratus (Winkler), en que la corona posee bordes afilados, finamente aserrados y en vista de perfil la cara interna del diente es más recta que en I. trituratus (cf. Ledoux, 1972).
Ledoux (1972) señala que una de las características dentales que muestra una tendencia evolutiva del género Isistius es la migración en la raíz del ojal, adquiriendo posiciones cada vez más bajas en su cara labial. Otro caracter que ha variado con el tiempo está representado por un aumento de la longitud de la raíz. A estos debemos añadir la variación morfológica de la corona que en las formas actuales es muy elongada.

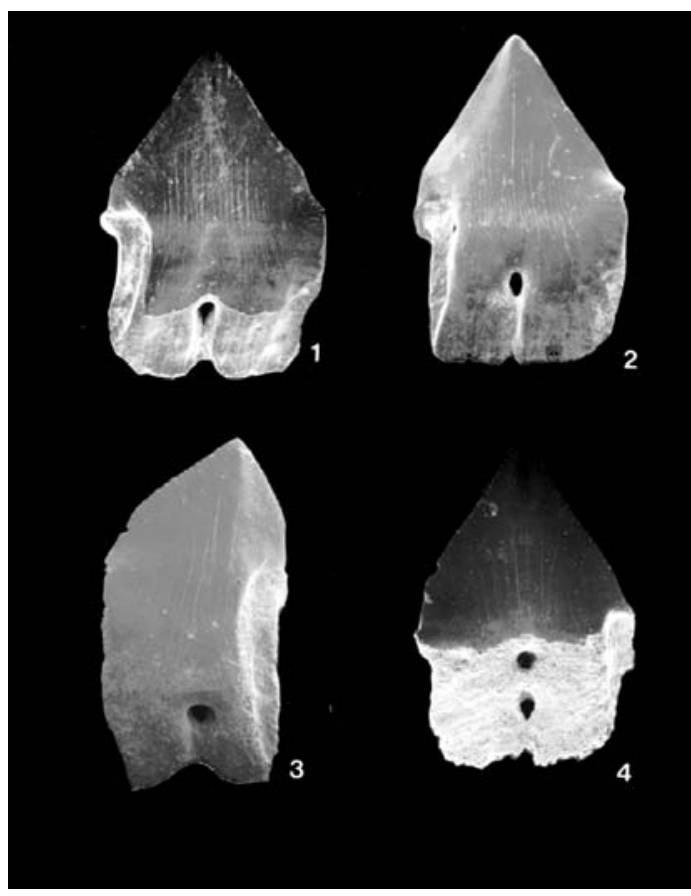

Lamina 1: 1: especímen CLM-019(8X) y 2: especímen CLM021(10X), dientes inferiores derechos anterolaterales en vista labial; 3: especímen CLM-026(15X), diente comisural inferior izquierdo, en vista labial y 4: CLM-022(10X), diente inferior derecho anterolateral en vista lingual.

\section{CONCLUSIONES}

El primer registro fósil de Isistius triangulus (Probst, 1879) en Costa Rica, corresponde al segundo hallazgo de ésta especie en América. Ambos hallazgos están relacionados a depósitos con asociaciones de seláceos de ambientes pelágicos medios y del batial superior. Sin embargo, aún faltan realizar los análisis batimétricos de la fauna de foraminíferos bentónicos, para obtener así una visión de conjunto. 
Por otra parte, la edad obtenida para este afloramiento específico es Mioceno Superior cuspidal-Plioceno Inferior basal, zona N-17-19 de foraminíferos plantónicos (límite MessinianoZancleano).

\section{AGRADECIMIENTOS}

Al Dr. Miguel Telles Antunes del Centro de Estratigrafía y Paleobiología de la Universidade Nova Lisboa y a la Licda. Maria Judite Matias, coordinadora del Centro de Documentación Científica y Técnica del Instituto Geológico e Mineiro de Portugal, por su valioso aporte bibliográfico. A la Licda. Diana Pizarro del Laboratorio de Micropaleontología de RECOPE, por la datación de las muestras y sus valiosos comentarios. Al técnico Eduardo Pérez Gamboa de la Dirección de Geología y Minas, por su asistencia en las labores de documentación, campo y laboratorio. Al técnico Rafael Loáciga de la Unidad de Microscopía Electrónica (U.M.E.) de la Universidad de Costa Rica, por la realización de las imágenes de barrido.

\section{BIBLIOGRAFÍA}

AGUILAR, T., 1993: Paleocología de Alto Guayacán, Formación Uscari Mioceno Provincia de Limón, Costa Rica. - Rev. Geol. América Central, 16: 51-60.

ANTUNES, M.T., 1972: Les squales (Crétacé et Tertiaire): intéret pour la stratigraphie et sa problématique. Mém. B.R.G.M. N. 77: 345-355.

ANTUNES, M.T. \& JONET, S., 1970: Requins de 1'Helvétien supérieur et du Tortonien de Lisbonne.- Rev. Fac. Cienc., Univ. Lisboa, 2. Ser. C (cienc. Natur), 16 (1): 119-280.

CAPPETTA, H., 1970: Les sélaciens du Miocène de la region de Montpellier. - Paleovertebrata, Mém. extraordinaire 1970: 1-139.

CAPPETTA, H., 1987: Handbook of paleoichthyology, Vol. 3B Chondrichthyes II. Mesozoic and Cenozoic Elasmobranchii. - Gustav Fischer Verlag, New York, 193 págs.

CAPPETTA, H., GRANIER, J. \& LEDOUX, J.C., 1967: Deux faunes des Sélaciens du Miocene méditerranéen de France et leur signification bathymétrique. - C.R. somm. Soc. géol. Fr., 7: 292-293.

CAPPETTA, H. \& LEDOUX, J.C., 1970: Comparaison de la faune ichthyologique miocene avec actuelle de Méditerranée. - Journées icththyol., 21-23.- Rome, C.I.E.S.M.

CASIER, E., 1958: Contribution à l'étude des Poissons fossiles des Antilles. - Mém. Suisses de Paléontolgie, 74: 95 págs.

CASIER, E., 1966: Sur la faune ichthyologique de la Formación de Bissex Hill et de la série océanique, de l'Ile de la Barbade, et sur l'âge de ces formations. - Eclogae geologicae Helvetie, 59(1): 493-515.

COMPAGNO, L.J.V., 1984: FAO species catalogue. Vol. 4 Sharks of the world. An annotaded and ilustrated catalogue of sharks species known to date. - FAO fish. Synop., 125(4), Part. 1: 1-249; Part. 2: 251-665.

GARRICK, J.A.F. \& SPRINGER, S., 1964: Isistius plutodus, a new squaloid shark from the Gulf of Mexico. - Copeia 1964, 4: 678-682.

GILLETTE, D.G., 1984: A marine ichthyiofauna from the Miocene of Panamá, and tertiary caribbean faunal province. - En: MacFadden, B.J. (ed.): Origin and evolution of the Cenozoic vertebrate fauna of Middle America. - J. Vertebrate Paleontology, 4(2): 172186.

KOZAREK, R.J. \& ORR, W.N., 1980: Ichthyoliths, Deep Sea Drilling Project Legs 51 through 53. - En: Init. Rep. DSDP, 51, 52, 53(2): 857-895.

LEDOUX, J.C., 1972: Les Squalidae (Euselachii) miocènes des environs d'Avignon (Vaucluse).- Documents Lab. Géol. Fac. Sci. Lyon, Notes et Mém., 52: 133175.

LONGBOTTOM, A., 1979: Miocene shark's teeth from Ecuador. - Bull. Brit. Mus. Natur. Hist. (Geol.), 32(1): 57-70.

SERRALHEIRO, R., 1954: Contribução para o conhecimento da fauna ictiológica do miocéno marinho de Portugal Continental. - Rev. Faculd. Ciênc. de Lisboa, C, 4(1): 39-119.

STRASBURG, D.W., 1963: The diet and dentition of Isistius brasiliensis, with remarks on tooth replacement in other sharks. - Copeia, 1963, 1: 33-40.

ZBYSZEWSKI, G. \& MOITINHO D'ALMEIDA, F., 1950: Os peixes miocénicos portugueses. - Comn. Serv. Geol. de Portugal, XXI: 309-412. 